other or to the ace and conld be returned without dificulty into the cavity of the abdomen. The right lung was much smaller than usual. The stomach contained food he had recently taken; the internal membrane of the left extremity was highly inflamed, probably from the operation of the emetic, and appeared to be the immediate cause of death.

A case of fatal hernia of some of the abdominal viscera, strangulated in the cavity of the thorax, is recorded by Dr. Clarke, in the Transactions of the Society in London for Medical and Chirurgical Improvement. The whole of the small and part of the great intestines had passed through on aperture in the diaphragm into the cavity of the chest. This aperture, which probably had existed from birth, was on the left side, about three inches from the ribs, more forwards than the natural opening for the osophagus. A portion of the epiploon was carried up with the colon, and also a part of the outer edge of the left lobe of the liver. There was very severe inflammation, producing symptoms of strangulation.

Norwich, February 15:5. 1

\section{PARTURITION FOLLOWED BY INVERSION OF THE UTERUS.}

By W. H. BORHAM, Esq.

Amove the most dangerous and distressing cases which occur to the accoucheur, perhaps none are so alarming as those where inversion of the uterus follows labour. Such events are happily very rare indeed; two only having been seen by Dr. Denman, one by the late Dr. Merriman, and one by the present Dr. Ramsbotham in a recent state whilst the patient was alive. Hence the narration of the following case, which occurred in my practice, will, I trust, plead a sufficient excuse for my giving it publicity. It also may be of importance to those who are engaged in collecting midwifery statistics.

Case. Mrs. H., aged 25, residing near Paddington Green, a fine strong woman, was delivered of her first child with the funis twice twisted round the child's neck, on January 31 st at 3 P.M., by Mrs. Collen of Paddington Street, a midwife of considerable experience, after a natural labour of sixteen hours' duration. The pains occurred at rather longer intervals than usual, and were very forcing. About an hour after the child was born, I arrired in consequence of a hasty summons, when the patient presented the following state. The whole of the uterus, dragging a small portion of the posterior part of the vagina with it, was lying (like a scrotal hernia) without the os externum; it was covered with the deciduary and other membranes, and the placenta was entirely attached to its fundus. The patient was lying upon her back, with her knees bent up. She was pulseless, had difficulty of breathing, was cold, prostrated, and exhausted; in fact, in a state of collapse. The uterus had been exposed for three-quarters of an hour, and had become much contracted. I immediately detached the placenta by inserting the index finger of my right hand between it and the uterus, and then peeled off the membranes attached to it (those which spring from the placenta). The midwife now well greased my left arm and hand, with which I grasped the uterus, and returned it within the vagina. I then withdrew my hand a little, formed my fingers into a cone, reintroduced it, gently carrying the fundus before me until I had carried it up to a level with the patient's umbilicus, when I had the satisfaction of finding that the uterus had righted itself into its normal position in the pelvis. Its neck and os then commenced a gradual contraction on the fingers, when I carefully and steadily withdrew my hand, desiring the midwife at the same time to make pressure just above the pubes with her hand. After the withdrawal of mine, I had a saucer placed upon the lewer part of the abdomen, and a bendage bound tightly it.
During this time, which lasted about fifteen minutes, the patient was in a state of great exhaustion, and it was only by the aid of brandy that I could at all arouse her. I romained with her an hour, by which time she rallied considerably. I then left, prescribing for her a mixture of sulphuric acid, sulphuric ather, and camphor mixture, every two hours.

At ten o'clock I wes again sent for, as a return was feared; but, on examining, I found it was only a clot escaping, and I placed a sponge in the vagina.

Feb. 1st. The patient remained in a very low state; the pulse was 150 , and feeble; she was very thirsty ; tho discharge was moderate. She was ordered to continue the mixture.

Feb. 2nd. The pulse was 120. The patient was very excited, with peritonitis threatening. I ordered two grains of opium and five of calomel to be taken directly, and a saline mixture with prussic acid. The patient had a desire to micturate, and for the first time I desired her to be moved for that purpose, when she passed a great quantity of urine. She felt exhausted, but much relieved.

Feb. 3rd. The pulse was 110. She had had four very liquid stools in the night which produced faintness. She complaincd of much pain in the bowels, and was restless. The tongue was furred; there was a dragging or tearing pain in the bowels; the discharge was offensive and purulent. I ordered fifteen leeches to be applied to the epigastrium, and a poultice afterwards; I also prescribed grey and Dover's powders to be taken every three hours, and an effervescing saline draught with an excess of ammonia and prussic acid every four hours.

Feb. 4th. She was better in every respect. The medicines were continued.

Feh. 5th. She was progressing favourably.

She continued daily to get better. Her milk came at the ninth day; and I left her convalescent at the end of three weeks.

Remarks. The midwife assured me that she did not use the slightest traction at the funis after the birth of the child, but that the uterus and attached membranes camo out all at once with one pain about half-an-hour after the child was expelled; and she thinking it a "tumour" desired I might be instantly sent for. The funis being twice convoluted round the child's neck greatly reduced its remaining length, and doubtless this circumstance assisted considerably in dragging down the fundus, particularly as the pains were described as "very forcing"; the fundus thus becoming cup-shaped from unaroidable traction, the whole uterus soon became inverted and expelled.

There is a division of opinion as to the advisability of detaching the placenta from an inverted womb before returning it. Much must of course depend upon the contracted state of this organ; the length of time it has been exposed, etc.; but I think the placenta should always be separated before returning the uterus, if the latter be in a contracted state; for the less the substance to be returned the easier is it accomplished. Nor need we fear hæmorrhage, for the uterus being inverted would drag down the uterine arteries into its inverted cavity; they would become intussuscepted, the uterus contracting upon them, forming an artificial tourniquet; and thus the supply to the uterine sinuses would be cut off (as was the case in this instance, for there was no hamorrhage during the operation of detachment). I therefore cannot think with some authorities that the patient is sure speedily to die of profuse hæmorrhage if the placenta be detached. On the other hand, if the uterus were in a very relaxed state, and its os well dilated or easily dilatable, the hæmorrhage might indeed be great if the placenta were partly detached; and in such a state there would be little difficulty in returning the whole mass, and perhaps it would be advisable to do so. But in a case lite my own it was desirable to return the uterus with as little addition to it as possible, owing to the contracted state of that tissue.

That the uterus should be immediately returned thare cannot be tro opinions; or its conteraction would soon pin- 
vent its return. The diminished size of the 08 would prevent the fundus from returning through it to its normal position, and strangulation would ensue, with its evil consequences. That peritonitis ensued in my case is not to be wondered at, considering how the peritonæum must have been interfered with by such a sudden revulsion of its tissue. The favourable termination of the case will certainly add a good practical confirmation to the opinion of those who are in favour of removing the placenta before returning the uterus. The uterus did not return with a sudden jerk, such as is asually ascribed to such cases.

Cambridge Terrace, Hyde Park, February 22nd, 185.

\section{SCARLATINA ANORMA FATAL BY INFLAM- MATION OF THE JUGULAR VEINS AND LATERAL SINUSES.}

By JOHN COCKLE, M.D., Physician to the City Dispensary, etc.

FOR some time past, scarlatina has been unusually prevalent, more especially in the neighbourhood of Lambeth Hill. Some of the cases observed were unattended by eruption, or, if any, of a very evanescent character. The morbid poison appears to have restricted its action principally to the salivary glandular system and mucous surfaces of the eye, nose, and ears, and by the nature, violence, and extent of its action, inducing inflammation of the jugular veins and lateral sinuses of the brain, such inflammation destroying life in its earliest or exudative stage. The following cases illustrate such an action of the poison upon the structures adverted to.

CASE I. A strong and healthy boy, aged 5 years, residing in Moor's Court, after feeling cold and ill, speedily became hot and restless. He then complained of sore throat and headache. Upon the following day, a scarlet eruption upon the body was observed by his mother, which, after a few hours, entirely disappeared, and was not again visible. The boy was delirious during the first two or three nights of his illness. The swelling of the throat gradually increased, and excessive external tumefaction was manifest, particularly over the region of the parotid glands. The face also was moderately swollen. A copious yellow purulent discharge was continually flowing from both nostrils, as also in smaller quantity from the eyes and ears. At no period of the child's illness was there any marked difficulty in swallowing, or evident embarrassment of the respiratory function. Neither cough nor vomiting existed. No false membrane could be detected lining either the fauces or nares. After the first three or four days the febrile heat subsided, and the pulse did not much vary from the normal type. At this period the child seemed unusually drowsy. Upon the fifth day, well marked coma set in, which terminated life upon the second day from its commencement. No examination of the body could be obtained in this case. A few days subsequently, the mother of this child was attacked with severe rigor, followed by considerable febrile reaction, transient scarlet eruption, excessive pain and swelling of the throat, followed by abscess of the tonsil. She speedily recorered; but the attack was attended with desquamation of the cuticle.

Case 1r. Within a day or two from the death of the above mentioned child, a remarkably fine and healthy male infant, aged 15 months, residing in the adjoining street, was seized with precisely similar symptoms. The same febrile stage and transient redness of the surface were observed. The parotid and neighbouring glands were greatly swollen, particularly upon the right side. Copious purulent discharge was constantly flowing from both the nostrils, and to a less extent, from the eyes and ears. The child apparently suffered considerable pain. The tumefaction and extreme pallor of the face contrasted with the bright red circle around the eyes; and the red and swollen notril imparted singularly striking expression to the child's physiognomy. The pupils were moderntely dilsted throughout. A greater difficulty of swallowing existed in this case; but this difficulty, as also the feverisin symptoms, soon subsided. There was no difficulty of breathing or other form of pulmonary disorder noted. The urine was remarkably diminished in quantity; but although carefully examined daily, gave no indication of albumen. Lithates only in excess were found. At one period the symptoms gave some promise of amendment; but suddenly the discharge from the nostrils acquired a remarkable fetidity. The child became gradually comatose, and soon after died in convulsions.

The post mortem examination was made by Mr. Selwood, about thirty hours after death. The body was considerably emaciated; the right parotid gland still remained greatly swollen; the eyes were much shrunk in the orbits, more particularly the left eye, which had lost nearly two-thirds of its original size; the pupils remained of the same size as observed during life: pus was still flowing from the right ear. Upon opening the skull, the dura mater was found apparently in its natural condition. Slight subarachnoid gelatinous effusion, most probably not inflammatory, existed both upon the anterior surface of the brain posteriorly, as also at the base, in the neighbourhood of the sylvan fissure. Moderate effusion existed in the lateral ventricles, and at the base of the skull free effusion to the amount of about an ounce. Slight softening was believed to exist in the neighbourhood of the fornix. Intense and most remarkable venous congestion was obvious both upon the anterior and posterior surfaces of the brain. In one vein, at the posterior part of the upper surface, in a portion of the canal, a straw-coloured substance resembling pus about an inch in length occupied the canal, but which, upon opening the vein, proved to be a colourless coagulum unattached, and which was readily drawn out entire. The vein still retained its natural transparency. Cpon opening the lateral sinuses and jugular veins, they were found red and opaque in places, and somewhat spongy and thickened, and completcly filled with a fibrinous coagulum slightly adherent to the walls, but readily extracted entire. The coagulum extended some distance beyond the apparently inflamed surface of the canal. Upon a microscopic examination, the coagulum was found to consist of amorphous fibrin; no pus-corpuscles could be detccted; neither could any concentric layers be recognised.

REMARKs. These cases, except in the absence of pustules, greatly resemble in many of their features the doscription given of glanders. Indeed, so much so, that at first I hesitated in the diagnosis ; and it is worthy of mention, that upon inquiry, I found the father of the first child was a carman, and in the habit of taking the child among the horses; and that just at the back of both the houses are stables containing a large number of horses, though it is certain the last child had not been inside them. I was also told there had been much sickness among these animals; but upon making an inquiry at the stables, I found this was denied, but whether justly or otherwise must be left. However this may be, this form of disease is exceedingly rare, whether regarded as a concomitant of scarlatina or even of inflammatory coryza. Death seems to have been the direct result of the obstruction offered to the return of the venous blood; inasmuch as fatal coma appears to hare been at once induced, without giving time for the symptoms of pyæmia to develop themselves.

It may be matter of opinion whether the phlebitis originated in the course of the canal, and by its extension induced coagulation of the blood secondarily; or, whether, from the large amount of surface involved and from chomical and atmospheric changes in the secreted pus, some poisonous matter was passed by endosmose into the veins, coagulating the blood primarily, and subsequently developing the phlebitis (septic phlebitis of Rokitansky.) I $\mathrm{em}$ inclined to believe this latter view the more correct one, inasmuch as in ordinary cases of scarlatina, where the parotid and submaxillary glands are greatly inflamed and eren suppurated, such inflammation of the large venous 